\title{
El principio de la autonomía del paciente pediátrico en estados de
} intersexualidad

The principle of the autonomy of the pediatric patient in intersex states

\author{
Luis Leonardo Macías-Solís \\ luis.macias.85@est.ucacue.edu.ec \\ Universidad Católica de Cuenca, Cuenca \\ Ecuador \\ https://orcid.org/0000-0002-0029-8201 \\ Selene Piedad Montoya-Chacón \\ selene.montoya@ucacue.edu.ec \\ Universidad Católica de Cuenca, Cuenca \\ Ecuador \\ https://orcid.org/0000-0001-6024-6449
}

Recepción: 15 de septiembre 2021

Revisado: 25 octubre 2021

Aprobación: 15 de noviembre 2021

Publicación: 01 de diciembre 2021 


\title{
RESUMEN
}

El desarrollo del presente artículo se enfoca principalmente en analizar la relación que existe entre el principio de autonomía del menor y los actos de salud que conlleven la figura del consentimiento informado o legítimo, los tratamientos y según las indicaciones médicas de la intervención quirúrgica para reasignación de sexo. Específicamente en la materialización del consentimiento informado, con el fin de establecer su influencia en el respeto del interés superior y de los derechos del paciente pediátrico. Por tal motivo se cuenta con fundamentos teóricos que detallan la información de cada variable, como son: Principios de autonomía, estados intersexuales y consentimiento informado; con lo cual se expone como se relacionan todas estas variables para darle coherencia y pertinencia al trabajo, a través de la investigación bibliográfica; proporcionando datos debidamente interpretados y analizados; constituyéndose en las bases y el sustento necesario para realizar las conclusiones.

Descriptores: Derechos civiles; igualdad de oportunidades; política de la salud. (Palabras consultadas del Tesauro UNESCO).

\begin{abstract}
The development of this article is mainly focused on analyzing the relationship that exists between the principle of autonomy of the minor and the health acts that entail the figure of informed or legitimate consent, the treatments and according to the medical indications of the surgical intervention for reassignment of sex. Specifically in the materialization of informed consent, in order to establish its influence in respecting the best interests and rights of the pediatric patient. For this reason, there are theoretical foundations that detail the information on each variable, such as: Principles of autonomy, intersex states and informed consent; with which it is exposed how all these variables are related to give coherence and relevance to the work, through bibliographic research; providing properly interpreted and analyzed data; constituting the bases and the necessary support to carry out the conclusions.
\end{abstract}

Descriptors: Civil and political rights; equal opportunity; health policy. (Consulted words of the UNESCO Thesaurus). 


\section{INTRODUCCIÓN}

Uno de los principios fundamentales del ser humano es la autonomía, la misma que le permite tomar decisiones, haciéndolo responsable de las consecuencias que ellas implican; pero hasta qué punto este principio tiene relevancia en las conductas y decisiones de los pacientes pediátricos, así como cuales son los parámetros 0 lineamientos que nos permiten determinar la pertinencia de la autonomía en estos pacientes, principalmente en la conducta médica multidisciplinaria del estado intersexual dentro del marco de la bioética y la normativa vigente, incluyendo el consentimiento informado.

Es claro, que es importante impulsar la autonomía en los niños, enfocarse en el respeto por la toma de decisión y los retos que conlleva la atención médica en pediatría. Sin embargo, la importante razón de determinar la edad en que puede determinarse que la autonomía se encuentra desarrollada con el fin de que pueda respetarse las decisiones de los niños es unos de los puntos seriamente a destacarse. Por otro lado, determinar cómo influye esta autonomía pediátrica en la relación clásica médico-paciente, que se vería reemplazada por un tridente de actores (paciente pediátrico, pediatra y padres) y con base en protocolos y normas garantizar la eficacia o resultado de un acto médico. Actualmente, en nuestro medio no existe una norma que permita verificar los principales inconvenientes que nacen con el principio de la autonomía y las dificultades que este conlleva con el paternalismo, llevado a cabo por la autoridad del pediatra, así como la de los padres. La existencia de una normativa clara permitiría contar con una base para el diálogo y la relación interdisciplinaria entre los actores, como médicos especialistas, pediatras, padres, incluyendo los mismos pacientes pediátricos, lo cual permitirá evaluar con un sentido crítico aquellas circunstancias contextuales, conceptuales y socio-éticas (Vázquez-Quezada et al. 2020).

En definitiva todas estas variables: Principios de autonomía desde varios puntos de vista, estados intersexuales, dimorfismo sexual y consentimiento informado se verificaran a través de una investigación bibliográfica y documental, tomando como fuentes: libros, revistas, páginas web, tesis, entre otros; teniendo así las bases y el 
sustento para la pertinencia del presente trabajo, realizado desde el tipo descriptivo, ya que a través de diferentes fuentes de información se pretende obtener resultados que describan el comportamiento y relación de los objetos de estudio.

Con base en lo expuesto, es importante recalcar el análisis realizado por Martha Tarasco renombrada investigadora de la Universidad Anáhuac de México Norte, quien establece que el acto de decidir un acto médico debe ser casi estrictamente, según la Bioética, realizado entre el médico y paciente, tomando en consideración que el galeno es quien posee la experiencia y el conocimiento profesional, pudiendo con esto proporcionarle al paciente la información y orientación necesaria sobre su enfermedad, los actos médicos a realizar y hacerle conocer sus beneficios y riesgos. Sin embargo, también enfatiza que hay decisiones que por su complejidad científica debe ser tomada por el médico.

\section{MÉTODO}

Con el fin de cumplir los objetivos se realizó una investigación descriptiva documental mediante revisión bibliográfica sustentada en métodos generales de la investigación científica, el exegético, revisión bibliográfica y analítico-sintético. Es así que mediante la exegética permitió el análisis de las leyes y normativas vigentes con el fin de llegar a entender de manera coherente; específicamente a las que se relacionan al principio de autonomía y que se encuentran en la Constitución Ecuatoriana, Código de la Niñez y la Adolescencia, y demás normas vigentes en nuestra legislación y organismos internacionales. Mediante la revisión bibliográfica y su análisis se pudo conseguir la interpretación de las fuentes científica consultada, tomando en consideración la actualidad y cientificidad.

\section{RESULTADOS}

\section{La intersexualidad y su incidencia en los pacientes pediátricos. Dimorfismo sexual, estados intersexuales.}

En este sentido, (Torrentera, 2019), define como intersexualidad o ambigüedad sexual, como aquel individuo con discordancia entre la morfología de los genitales 
externos, como consecuencia de errores en el desarrollo sexual del feto, ya sea por alteraciones cromosómicas, de carácter embriológicas o por defectos bioquímicos. En términos generales, la diferenciación sexual requiere de una serie de procesos que deben estar perfectamente emparejados en el cual interviene una gran cantidad de genes en la formación y síntesis de enzimas, hormonas y factores importantes, las anomalías que se pueden presentar en cada una de las etapas del desarrollo fetal, conlleva a errores o desórdenes de diferenciación sexual, denominados comúnmente como estados intersexuales.

Lamentablemente en nuestro país no contamos con datos estadísticos sobre casos de estados intersexuales, sin embargo según la literatura médica la incidencia de intersexualidad en pediatría depende principalmente del tipo de alteración en el desarrollo de la gónada fetal o disgenesia gonadal, especialmente aquellos casos en donde las gónadas son sustituidas por cintillas gonadales (tejido fibroso), es así que la Sociedad Española de Endocrinología Pediátrica identificaron la incidencia de los estados intersexuales, que de manera general se encuentran en el $2,5 \%$ de adolescentes de ambos sexos de una población normal. Sin embargo, según la etiología y los síndromes identificados se pueden encontrar las siguientes incidencias: el Síndrome de Turner se presenta aproximadamente de una de cada 2.000 mujeres recién nacidas; el Síndrome de Klinefelter presenta una incidencia de uno o dos por cada 1000 varones; el Síndrome de Kallman, con una incidencia difícil de calcular se estima en uno por cada 10.000 a uno por cada 86.000 personas.

Es importante señalar, que existe en algunos adolescentes de ambos sexos un retraso puberal fisiológico, conocido como retardo constitucional del crecimiento y pubertad con una incidencia de $1-2,5 \%$ de la población general, el cual debe considerarse en los diagnósticos diferenciales de los estados intersexuales y como la forma más benigna de los mismos. Existen tres etapas de diferenciación sexual los cuales son el sexo genético, gonadal y el sexo genital o anatómico, sin embargo, durante el desarrollo de una persona de cualquier sexo se adquieren caracteres sexuales secundarios, para lo cual se podría añadir el sexo fenotípico, sexo social y psicosexual. 
Inmediatamente después de la fecundación se produce las cascadas de procesos de diferenciación sexual que conlleva a la clasificación de sexo masculino y sexo femenino, es en este momento que se determina el sexo genético. Aproximadamente hasta la quinta semana de vida fetal los genitales son idénticos en ambos sexos, sin embargo, desde la sexta semana aproximadamente 30 genes que se encuentran localizado en los brazos de los cromosomas $X$ y $Y$ se activan para intervenir en la diferenciación sexual, sin embargo, es partir de los 3 meses de vida fetal que se empieza a identificar en el sexo femenino el ovario y entre las 6 y 7 semanas se identifica en el sexo masculino, el testículo.

Cualquier anomalía o error en cada uno de los procesos de la vida fetal de diferenciación sexual conlleva a alteraciones importantes de carácter estructural y funcional de los genitales externos, lo que se conoce como estados intersexuales. La literatura médica menciona cuatro trastornos que se presentan o no con ambigüedad sexual al momento del nacimiento, estableciéndose el estado de intersexualidad:

a. Pseudohermafroditismo femenino. O llamado también virilización femenina, que cuenta con una característica genética de $46 \mathrm{XX}$, además de genitales internos con características normales, pero se asocia a ciertos grados de ambigüedad genital: clitorimegalia; formaciones labioescrotales; seno urogenital independiente o la existencia del mismo, pero además se comprueba la no presencia de gónadas en la zona labio-escrotal y en la región (canal) inguinal.

b. Pseudohermafroditismo masculino. Caracterizado principalmente por presentar sexo gonadal y genético de origen masculino, sin embargo, los genitales presentan características femeninas o presentarse cierto grado de ambigüedad sexual.

c. Hermafroditismo verdadero. Caracterizado por que las gónadas contienen tejido o parénquima tanto testicular como ovárico. Estos individuos contienen ambigüedad de genitales internos y de los genitales externos.

d. Disgenesia gonadal. Son aquellos, en donde en las gónadas no se aprecian las típicas células germinales, ni cualquier otro elemento de esta vía germinal. 
La disgenesia gonadal suele asociarse principalmente al cese de migración de las células germinales primitivas hasta las gónadas indiferenciadas o a la involución de las mismas una vez han llegado a estas últimas.

\section{Tratamiento de los estados intersexuales.}

En la actualidad existe un gran avance en el tratamiento de los estados intersexuales en los campos de manejo de los estados hormonales, genética y biología molecular cuya finalidad estará centrada principalmente de forma progresiva en la maduración general corporal, genital y psicológico, sin embargo, todavía sigue siendo una gran problemática para los profesionales de la salud encontrarse con recién nacido que presentan genitales ambiguos, lo que conlleva a que se formen equipos multidisciplinario de salud y se establezcan protocolos basado en realizar un diagnóstico y tratamiento más temprano en la infancia. No obstante, existe cuestionamiento del tratamiento específicamente quirúrgico en fase temprana de la infancia debido a que no se puede obtener un consentimiento informado o legítimo a tales pacientes.

El tratamiento quirúrgico de un paciente pediátrico en estado intersexual se considera un gran desafío para el médico, principalmente por la gran cantidad de técnicas y las decisiones que deben tomarse. De manera general, la conducta actual ante un paciente con síndrome intersexual se le realizan estudios cromosómicos, hormonales y anatómicos y con esto se define la asignación de sexo mediante cirugía temprana (aproximadamente antes de los 18 meses), recordando que las tomas de decisiones siempre van a estar en dependencia con la complejidad del trastorno que origina el estado intersexual.

Sin embargo, en la actualidad existen grupos de pacientes adultos no conformes con el tratamiento quirúrgico recibido de forma temprana, se ha conformado una sociedad denominada ISNA que por su siglas ingles se denomina Intersexual Society North América, quienes no están de acuerdo con la realización de cirugías temprana, especialmente de tipo cosmética, tomando en consideración que a edades tempranas de la vida no existe la madurez cognitiva suficiente para la toma de decisiones y poder 
ofrecer un consentimiento legítimo, para lo cual proponen una conducta terapéutica que consiste.

Una vez identificado un recién nacido con genitales ambiguos éste es abordado por un equipo multidisciplinario en conjunto con los padres, a estos pacientes se los someten a estudios cromosómicos, hormonales y anatómicos para luego tomar dos vías: la primera que consiste en asignación del género y nombre del paciente con posterior seguimiento por el equipo multidisciplinario en conjunto con los padres, y la segunda vía, consiste en esperar que el paciente cumpla con la madurez cognitiva necesaria para tomar la decisión del sexo que desea con cirugía correctora, que para algunos autores según estudios realizados podría partir desde los 12 años y luego continuar con el seguimiento hasta la adultez.

\section{El principio de la autonomía en la bioética y pediatría.}

Uno de los mayores inconvenientes en la conceptualización del consentimiento legítimo o informado, es sin duda la aplicación del principio de autonomía de los pacientes pediátricos, los mismos que presentan muchas limitaciones en su inclusión al momento de decidir por los actos de salud a realizarse, ya sea de manera asistencial o en temas de investigación. Sin embargo, en la actualidad existe un cambio progresivo del modelo de relación médico y paciente, el mismo que empieza a darle mayor importancia a eliminar o disminuir el paternalismo y se enfoca en una mayor participación de manera consciente e informada en los escenarios de tomar decisiones, tomando como punto central la familia, retirando el concepto de que el paciente pediátrico es incapaz físico y moral de tomar decisiones, dándole la característica de ser "propiedad del representante legal", favoreciendo de esta manera en la actualidad a que el pediátrico sea considerado como un individuo dinámico en el ejercicio de sus derechos, cuya autonomía, razonamientos y toma de decisiones se encuentran en un proceso de desarrollo continuo según su el grado de madurez de entendimiento.

Es así como actualmente, dada la evolución que existe en la percepción social y moral de los menores, a nivel general se impone un concepto con alto impacto jurídico que 
es el menor maduro o menor adulto, que son nada más que aquellos menores de edad conscientes del goce de sus derechos y tomas de decisiones, para lo cual debe de estar presente varias virtudes como la prudencia, la experiencia y la capacidad de discernir sobre los beneficios y riesgos de sus propias decisiones. Este concepto, se basa en diversas teorías que mencionan el proceso del desarrollo moral y cognitivo de los pacientes pediátricos, además de actuales estudios del desarrollo del razonamiento. Para comprender el desarrollo moral en los niños, citamos el modelo cognitivo de Piaget (1932), quien indica que dicho desarrollo atraviesa por cuatro etapas, según su grado de madurez:
a) Egocéntrica.
b) Autoritaria.
c) Reciprocidad.
d) Equidad.

Varios investigadores establecen que aproximadamente a los doces años de edad, se alcanzaría la etapa de equidad, teniendo un alto grado de madurez del desarrollo cognitivo desde los treces hasta aproximadamente los dieciochos años de edad. Este madurez moral y cognitiva es importante para discernir y ajustar el momento de tomar una decisión en los pacientes pediátricos con los requisitos fundamentales del consentimiento legítimo, específicamente con los puntos más abstractos de dicho consentimiento, tal y como lo afirman (Wendler \& Jenkins, 2008), los menores de edad dependiendo de su madurez cognitiva y/o moral primero desarrollan una comprensión completa de los hechos concretos del medio que los rodea, para luego poder discernir las partes abstractas, pudiendo así determinarse que una vez desarrollada las compresión abstracta del mundo, son individuos capaces de discernir la información y de manera autónoma tomar sus propias decisiones y por lo tanto brindar un consentimiento legitimo e informado.

Por otra parte, existe una propuesta de Kohlberg en (Díaz-Serrano, 2015), el cual determina que la madurez de razonamiento moral de los pacientes pediátricos se desarrolla en tres fases o niveles: 
a) Nivel preconvencional: en la cual el menor identifica las normas morales por medio de las figuras de autoridad, mediante actos de recompensa y castigo, identificando las consecuencias que surgen dependiendo de los actos realizados.

b) Nivel convencional: el razonamiento moral se adapta a las normas sociales aceptadas o aquellas que sirven para sanción, se asimila la ley y/o normativas como herramienta de regulación externa según los actos realizados.

c) Nivel posconvencional: se identifican los principios morales que ayudan para la creación de normas, pudiendo incluso hacer usos de las leyes para contradecir con sustento.

Además, Carol Gilligan establece que son múltiples las teorías propuestas al respecto del desarrollo del razonamiento moral y la evolución cognitiva del comportamiento, entre ellos los sentidos narrativos y hermenéuticos de la experiencia moral, la psicología con un enfoque social, el estudio del comportamiento por medio de la neurobiología, además de la sociología basados desde el punto de vista de la biología, las cuales han sido evidenciadas a partir de la evolución social de las personas, además de los diferentes estudios de imagen funcionales que existen en la actualidad (Chisari-Corti, 2018).

En conclusión, basados en las afirmaciones de varios autores según los resultados de sus estudios realizados y tomando en consideración diversas legislaciones en el mundo se ha definido como limite a los 12 años, edad en la cual se considera al menor con la capacidad suficiente de discernir información, así como determinar los riesgos y beneficios de las decisiones tomada.

Es importante indicar que desde la rama de la bioética se establece que la decisión médica debe comprender un vínculo entre el paciente y el médico, este último ofreciendo su experticia y aplicación de la lex artis en el acto médico, con el fin de dar a comprender los riesgos, las complicaciones, las posibilidades de éxito, beneficios de las decisiones tomadas y consecuencias $u$ opciones de realizarse o no el tratamiento. Sin embargo, en muchas ocasiones se anteponen decisiones en el campo 
de la medicina que exceden el conocimiento científico, de ahí que a la medicina no solo se la puede considerar como una ciencia, sino que además es un arte el cual requiere de valores éticos bien definidos. Se establece que, durante las diferentes fases madurativas cognitivas, se realizan en base a las cuestiones y juicios éticos.

Establecer lineamientos en donde el paciente pediátrico pueda tomar sus propias decisiones es poco aplicable a nivel jurídico sino se toma en consideración la maduración cognitiva del menor, ya que para la bioética, el resultado final de la decisión tomada siempre deber estar centrada en el bien del paciente, tomando en consideración que dichas decisiones podrían ser erróneas por parte del menor por varios factores entre ellos la falta de comprensión de la solución o del problema, de los riesgos y beneficios e incluso por los temores que se puedan presentar.

Sin embargo, como ya se mencionó anteriormente desde un punto de vista moral esto no significa que no se tome la importancia a la decisión del paciente pediátrico. En tales casos es beneficioso establecer y fortalecer mucho más la relación médica paciente, incluso sumándose a esta importante relación, con el fin de formar un tridente: médico - paciente - representante legal, con el fin explicar de mejor manera la condición del paciente y cada uno de los procedimientos a realizar. Por lo mencionado, brindar información y solicitar su colaboración al menor de acuerdo a su desarrollo cognitivo establecerá vínculos más estrechos sintiéndose parte del tratamiento con beneficios claros, incluso en el entendimiento, o no llegar a experimentar soledad.

\section{El consentimiento legítimo en el marco jurídico}

Establecer autonomía en los pacientes pediátrico conlleva establecer una relación en base a la verdad, el respeto de la privacidad mediante la protección de la confidencialidad, con el único fin de conseguir el consentimiento legitimo o el asentimiento. En la actualidad muchos autores siguen apoyando el principio aristotélico de que los niños no son aptos para tomas de decisiones, por su inmadurez y falta de prudencia. Sin embargo, en contraposición existen otros investigadores que sostienen la teoría de que los pacientes pediátricos logran identificarse con el principio 
de autonomía y equidad alrededor de los 12 años, e identifican el goce de sus derechos individuales y principios éticos, a partir de los 13 a 18 años, como se había mencionado anteriormente.

El consentimiento legítimo en pediatría es un proceso el cual permite la toma de decisiones dirigido específicamente en la formación de una relación en forma de tridente médicos-pacientes-padres o representante legal. Es una forma de asentimiento que va mucha más allá de la aplicación jurídica o netamente moral, sino que abarca un mecanismo de promoción, inclusión y aplicación del principio de autonomía del menor especialmente en lo que se refiere a las decisiones en los actos de salud.

En la actualidad, el consentimiento legítimo, las tomas de decisiones y la relación de autonomía y desarrollo cognitivo del paciente pediátrico, establecen dos situaciones: 1) de protección, a través de las obligaciones de los adultos hacia ellos; 2) del interés efectivo, que consiste en aplicar los derechos del menor como base legal para garantizar los intereses del paciente pediátrico.

El Código de Niñez y de Adolescencia del Ecuador establece la importancia del interés superior del menor en su artículo 1 que tiene como finalidad lograr el desarrollo integral del menor, así como el goce de los derechos dentro del contexto de libertad, dignidad y equidad. Además, en este mismo marco legal en su artículo 60 dispone el derecho del menor a ser consultado tomando en consideración en la medida de su edad y madurez. Así mismo, la Ley de Derechos y Amparo al Paciente indica en su artículo 6 el derecho de los pacientes en la toma de sus propias decisiones.

Además, la Constitución del Ecuador del 2008, dispone en su artículo 362 que los servicios de salud garantizarán la aplicación del consentimiento legitimo y/o informado, manteniendo la confidencialidad de la información. Mientras que la Ley Orgánica de Salud del Ecuador, promueve en su artículo 7 como derecho inviolable del paciente, mantener la autonomía a través del uso del consentimiento escrito, así como la toma decisiones respecto a su salud, excepto en casos de riesgo para la vida de las personas (urgencia y/o emergencia). 
Además, dentro de las normativas vigentes ecuatoriana existe un protocolo de gestión y de buena aplicación del consentimiento informado en las instituciones de salud, el cual fue suscrito mediante Acuerdo Ministerial № 00005316 por parte del Ministerio de Salud del Ecuador el cual define al consentimiento informado como una forma de comunicación que permite mantener la relación médico - paciente, mediante cual un paciente toma la decisión de forma voluntaria de un acto de salud a realizarse.

Esta misma normativa de aplicación del consentimiento informado o legítimo establece directrices para aplicar en pacientes menores de edad, la cual indica que deberá ser suscrito por el representante legal. Pero además indica, que solo en caso de padres menores de edad que se hayan emancipados, el consentimiento legitimo o informado podrá ser presentado por ellos mismos, tanto para actos de salud a realizarse de manera personal o a sus hijos. En este contexto es importante señalar que el Código Civil Ecuatoriano, en su artículo 28 indica que la madre, el padre que cuente con la patria potestad, así como un curador o tutor son considerados representantes legales de una persona cuando lo requiera, estos ultimo actúan en caso de ausencia de los padres. En caso de no existir representante legal de un paciente pediátrico, el galeno y/o profesional de la salud, sustentado bajo criterio médico, quien actuará siempre y cuando se imponga el beneficio del menor.

Por último, el mismo Acuerdo Ministerial 5316, en concordancia con lo establecido por varios autores tomando en consideración el desarrollo cognitivo del menor, la información a paciente pediátricos a partir de los 12 años, será brindada por el profesional de la salud de manera verbal, sin embargo, se mantiene la formulación del consentimiento informado o legítimo por escrito a los representantes legales. Con base a lo expuesto es importante recalcar que en Ecuador no existe totalmente una autonomía del paciente pediátrico en temas de salud como existe en otros países, por ejemplo, en España se sugiere legalmente la madurez desde los 16 años y por lo tanto podrá tomar la decisión ante un acto de salud.

En el Ecuador, los estados intersexuales son patologías que jurídicamente hasta el 2014 se la podría describir como huérfanas, ya que aquellos pacientes pediátricos que nacían con genitales ambiguos eran tratados solamente mediante el criterio médico, 
sin el consentimiento de los pacientes o de los representantes, tal y como se puede verificar en la Sentencia 056-2014, en la cual mediante un acto quirúrgico se asigna sin el debido consentimiento el sexo masculino a la menor quien responde al nombre de Asley, quién aproximadamente a los 15 años por un proceso de menstruación "interna" identifica su estado de intersexualidad. Para lo cual el médico indica que de acuerdo a su criterio era lo mejor para su hijo. En la actualidad, en los hospitales del Ecuador no existen protocolos médicos aplicados a la atención de pacientes intersexuales, sin embargo, se realizan varios procedimientos quirúrgicos mediante el consentimiento del representante legal, dejando a un lado la decisión del propio paciente, y por ende la perdida de autonomía.

Históricamente, el consentimiento informado nace en el año de 1957 en Estados Unidos a raíz de un famoso proceso judicial llamado caso Salgo, en donde el paciente demanda por negligencia al médico que le realizó un procedimiento llamado aortografía, en el cual se presentó como complicación del acto médico una parálisis permanente del paciente, para lo cual la Corte determinó que el galeno no informó sobre las complicaciones que se podrían presentar durante 0 después del procedimiento y con esto dar paso al consentimiento del paciente. Naciendo de esta manera la idea de que el consentimiento legítimo sea considerado un derecho del paciente. Como ya se estableció el consentimiento legítimo es un proceso mediante el cual se intercambia datos entre el personal de salud y paciente, el cual debe contar con varios elementos principales:

a) Información precisa: mediante la cual se da a conocer al paciente los actos de salud a realizar, además de explicar los riesgos y beneficios.

b) Aptitud: son aquellas habilidades que le permite al paciente poseer la capacidad de comprender y discernir los riesgos y beneficios de un acto médico, teniendo como resultado la libertad de decidir.

c) Voluntad: requisito importante al momento de toma de decisión frente a un acto médico, considerándose como un derecho del paciente el hecho de elegir. 
El asentimiento informado o legítimo se considera como un proceso que propone incluir a los pacientes pediátricos, principalmente en la libertad de tomar decisiones; aunque es un tema de amplia importancia en la bioética, en la práctica principalmente en nuestro medio aún es muy poco utilizado, a pesar de que organismos internacionales como la (UNICEF, 2006), donde se sostiene que la libre expresión del niño es un derecho personalísimo.

Desde el ámbito jurídico tanto el consentimiento y el asentimiento legítimo en pacientes pediátrico con estado intersexuales son mecanismos directos de protección de principios y derechos como la autonomía, dado que la toma de decisión en cuanto a la afinidad y la identidad sexual, como ya se determinó anteriormente en este trabajo con los estudios realizados por algunos autores, es un acto que se encuentra ligado directamente con la madurez de la personalidad del individuo. El tratamiento quirúrgico de los estados intersexuales en muchas ocasiones al no configurarse como una urgencia o emergencia, podrían convertirse en actos innecesarios e irreversibles al realizarse de manera temprana, siendo perjudicial a futuro el hecho de no tomar en consideración la voluntad del paciente provocándose problemas físicos, sociales y psicológicos, así como violación al derecho de la dignidad humana.

Es importante analizar que los tratamientos quirúrgicos tempranos en estados intersexuales vulneran directamente el derecho a la salud, tomando en consideración que afecta la integridad física y psicológica del paciente, pero además se alteran los derechos reproductivos futuros de los pacientes intersexuales. Por lo tanto, de manera general se evidencia una alteración de la personalidad al no permitir la decisión de su identidad sexual, vulnerando el interés superior del menor.

Es de conocimiento que en la aplicación del consentimiento legitimo los padres, curadores o tutores son considerados representantes legales, los mismos que podrán tomar decisiones en base a responsabilidades parentales, sin apartarse del deber de velar por el interés de su representado. Sin embargo, en algunos casos de intersexualidad al tratarse de cirugías y tratamientos irreversibles deberán tomarse en consideración al consentimiento del paciente pediátrico con el fin de garantizar el interés superior del menor como lo establece el artículo 6, del Convenio de Oviedo, 
el cual indica que la intervención de una persona incapaz de consentir o decidir, solo podrá realizarse cuando este acto médico redunde en su beneficio directo, de manera más específicas cuando este tipo de cirugía no son urgencias o emergencias y son consideradas cosméticas (Puig-Hernández, 2021).

Actualmente varios organismos internacionales que velan por los Derechos Humanos, entre los cuales se podría mencionar al Comité de Derechos de los Niños el Alto Comisionado de los Derechos Humanos de las Naciones Unidas, el Relator Especial para el Derecho a la Salud, Comité contra la Tortura, entre otros, se han pronunciado sobre los estados intersexuales, considerando a las problemáticas específicas de algunos países en cuanto a las garantías de derechos fundamentales de estas personas.

La resolución jurídica N²191 es una de las más relevante y completa realizada la Asamblea Parlamentaria del Consejo de Europa, dicha resolución fue elaborada en el año 2017, en la cual se indica las acciones o mecanismos pertinentes que podrían sumarse a las legislaciones de los diferentes países, con el fin de poder garantizar los derechos de individuos intersexuales.

La determinación legal del sexo y del género ha llevado a que se realicen varios estudios de carácter jurídico por organismos internacionales, entre ellos la Comisión Interamericana de Derechos Humanos que en el 2012 publica algunos términos sobre orientación sexual, la identidad de género y expresión de género, en dicho trabajo se incorpora los conceptos de Mauro Cabral, quien ya desde el 2005 define que una es persona intersexo y estandariza la variabilidad de la imagen masculina o femenina que se encuentra dentro de la cultura de la población.

Como ya se ha indicado el consentimiento informado es un elemento principal en el tratamiento de los estados intersexuales, es así que la Corte Constitucional Colombiana mediante Sentencia SU-337/99, en la cual se estableció una doctrina jurídica de la aplicación del consentimiento informado, la cual es pionera a nivel mundial frente al derecho de los intersexuales a la autodeterminación de la identidad sexual. Dicha sentencia, a la cual le antecede una acción de protección (tutela) realizada por la madre de una niña hacía el Estado de Colombia, luego de que el 
Seguro Social de este país se negara a realizar una cirugía de reasignación sexual a la niña, la cual había sido diagnosticada con seudohermafroditismo masculino desde los tres años de edad por un médico especialista, quien recomendó el tratamiento quirúrgico en aquel momento.

Unas de la causa principal por la que los médicos se negaron a realizar el acto quirúrgico, es el antecedente de ya existir en la Corte Constitucional de Colombia un dictamen jurídico (T-477) del año 1995 en la cual se dictaba que los actos médicos que afecten la identidad sexual del menor no podrán ser decisión de los padres o representante legal, debiendo ser una decisión exclusiva del paciente. Habiéndose tomado en consideración la gran cantidad de información sobre criterios médico y social sobre intersexualidad en el transcurso del juicio, esta Corte Constitucional, concluye que los actos médicos y/o quirúrgicos para lograr la reasignación del sexo, necesitan de un consentimiento legítimo e informado.

Además, el tribunal decide mediante fundamento legal $n^{\circ} 66$, que la Corte no evidencia motivos relevantes que puedan justificar la necesidad de urgencia de las terapias actuales para reasignación sexual, y que estos actos médicos establecerían riesgos importantes de daños irreversibles y que podrían incluso ser graves. De manera particular, durante el proceso se tomó en consideración la edad y madurez de la menor, por lo tanto, la Corte refiere sustentados en fundamentaciones jurídicas $n^{\circ} 88$ que los pacientes pediátricos aproximadamente a los cinco años de edad han desarrollado totalmente una identidad de su género, pero que además han logrado discernir el principio de autonomía y poder tomar sus propias decisiones con respecto a su cuerpo.

La Corte también deliberó uno de los requisitos del manejo del consentimiento legítimo, el cual es que debe de existir una decisión muy bien informada, dicha consideración se puede verificar en el fallo, para lo cual tuvieron como fundamento ( $\left.{ }^{\circ} 64\right)$ que a diferencia de años anteriores, hoy en día la información que se da a los padres es mucho más completa, pero sin embargo existen nudos críticos con respectos a los protocolos de actuación, entre los principales la premura con que los padres deben tomar una decisión a pesar del daño emocional y psicológico que 
presentan, sumado a esto que el personal médico no ofrecen medidas alternativas de tratamientos, no informan sobre los beneficios y los riesgos que se podrían presentar con la terapéutica actual, nudos críticos que sin duda alguna vician el consentimiento informado. Con base suficiente, debido a los principales fundamentos jurídicos la Corte Colombiana resuelve no dar paso a la reclamación mediante una acción de tutela (protección) realizada por la representante legal (madre) de la paciente pediátrica.

\section{CONCLUSIONES}

El principio de autonomía, toma de decisiones y consentimiento informado o legitimo se encuentra ampliamente legalizado en el Ecuador como un derecho que brindan los servicios de salud.

A pesar de que existen normativas que regulan el consentimiento legítimo y su aplicabilidad en los establecimientos de salud, se observa una deficiente manera de aplicación en pacientes pediátricos, en estado intersexual.

En nuestro país no existe estadísticas claras sobre pacientes con estados intersexuales, sin embargo, es conocido que en los establecimientos de salud se realizan intervenciones quirúrgicas a temprana edad con genitales ambiguos, omitiendo así el derecho a decidir sobre su propio cuerpo e incluso se hace caso omiso a las indicaciones que establecen la Comisión de los Derechos Humanos, que en el 2015 recomendó prohibir toda intervención médica en menores con síndromes intersexuales.

Es evidente que en el Ecuador la situación jurídica de los pacientes con estados intersexuales es escasa, por no decir nula, ya que no existe normativa alguna que regule los procedimientos y toma de decisiones de este grupo de paciente, con el fin de garantizar sus derechos dentro del ordenamiento jurídico de manera específica relacionado al ámbito de la salud.

Es realmente necesario que el Estado caracterizado por ser plenamente garantista, con el objetivo de disminuir aquellos eventos que violentan los derechos, principios de autonomía y toma de decisión de los pacientes menores en estado intersexual, 
establezca un régimen jurídico en el sector salud para garantizar la dignidad de este grupo de pacientes.

Uno de los principales problemas éticos de las intervenciones quirúrgicas de reasignación de sexo en pacientes pediátricos intersexuales es la aplicación del consentimiento legítimo y/o informado, pero además se suma la falta responsabilidad estatal al no establecer políticas públicas para la atención en salud de estos pacientes, para lo cual se podría tomar en consideración a las legislaciones de otros países que ya cuenta con decisiones más profundas al respecto, y poder ajustarlo a la realidad de la población ecuatoriana.

Las consecuencias jurídicas de la reasignación sexual están directamente vinculadas principalmente a la exclusión de derechos, sobre todo el de la libre decisión de la identidad sexual, por lo tanto, establecer normativas y bases jurídicas en la legislación del Ecuador mejorara en gran medida las garantías del derecho de los pacientes pediátricos en estados intersexuales.

\section{FINANCIAMIENTO}

No económico.

\section{AGRADECIMIENTO}

A las Autoridades del Programa de Posgrados de la Universidad Católica de Cuenca por permitir de la manera más cordial el desarrollo del presente artículo.

\section{REFERENCIAS CONSULTADAS}

Chisari-Corti, B. (2018). El desarrollo moral según Carol Gilligan. Estudio exploratorio en una muestra de mujeres y varones adultos de dos comunidades urbanas [Moral development according to Carol Gilligan. Exploratory study in a sample of adult women and men from two urban communities]. http://rpsico.mdp.edu.ar/handle/123456789/731

Código Civil Ecuatoriano. Codificación No. 2005010. Recuperado de https://n9.cl/x5ko

Código de la Niñez y la Adolescencia. Registro Oficial 737 de 03-ene.-2003 Ultima modificación: 07-jul.-2014. Recuperado de https://n9.cl/y13e 
Constitución de la República del Ecuador 2008. Registro Oficial 449 de 20-oct-2008 Ultima modificación: 13-jul-2011. Recuperado de https://n9.cl/hd0q

Corte Constitucional de Colombia. Sentencia T-477/20. Recuperado de https://n9.cl/d09fw

Corte Constitucional Colombiana. Sentencia SU-337/99. Recuperado de https://n9.cl/a1lw8

Corte constitucional del Ecuador. Sentencia No. 056-14-SEP-CC. Recuperado de https://n9.cl/agwmo

Díaz-Serrano, J. (2015). El desarrollo del juicio moral en Kohlberg como factor condicionante del rendimiento académico en ciencias sociales de un grupo de estudiantes de educación secundaria [Development of Moral Judgment according to Kohlberg as an Academic Performance Determining Factor in Social Sciences for a Group of Secondary Education Students]. Revista Electrónica Educare, 19(3), 72-85. https://dx.doi.org/10.15359/ree.19-3.8

Huertas Sepulcre J. (1979). Síndrome de Maestre de San Juan-Kallman-De Morsier. Un caso clínico [Maestre de San Juan-Kallman-De Morsier's syndrome. A clinical case]. Revista clinica espanola, 154(1-2), 63-64.

Ley Orgánica de Salud del Ecuador. Registro Oficial Suplemento 423 de 22-dic.-2006 Ultima modificación: 18-dic.-2015. Recuperado de https://n9.cl/4kfc

Piaget J. (1932). Le jugement moral chez l'enfant. Neuchâtel: Dclachaux \& Niestlé,

Puig-Hernández, M. (2021). Algunas reflexiones sobre el Convenio de Oviedo y los productos sanitarios. Responsabilidad y certificación en el caso de los cíborgs [Some reflections on the Oviedo Convention and medical devices. Liability and certification in the case of cyborgs]. Revista de Bioética y Derecho, (51), 299320.

Torrentera, A. (2019). Intersexualidad e intertextualidad. Sentido común y políticas corporales como desafío cultural [Intersexuality and intertextuality. Common sense and bodily policies as a cultural challenge]. Cuicuilco. Revista de ciencias antropológicas, 26(74), 87-104.

Trovó de Marqui A. B. (2015). Síndrome de Turner e polimorfismo genético: uma revisão sistemática [Turner syndrome and genetic polymorphism: a systematic review]. Revista paulista de pediatria : orgao oficial da Sociedade de Pediatria de Sao Paulo, 33(3), 364-371. https://doi.org/10.1016/j.rpped.2014.11.014 
UNICEF. (2006). Convención sobre los Derechos del Niño [Children's rights convention]. Recuperado de https://n9.cl/pnq7

Vázquez-Quezada, C., Narváez-Zurita, C., Trelles-Vicuña, D., \& Erazo-Álvarez, J. (2020). La tenencia compartida, alcances y su aplicación en el Ecuador [Shared tenure, scope and its application in Ecuador]. IUSTITIA SOCIALIS, 5(1), 474491. http://dx.doi.org/10.35381/racji.v5i1.626

Wendler, D., \& Jenkins, T. (2008). Children's and their parents' views on facing research risks for the benefit of others. Archives of pediatrics \& adolescent medicine, 162(1), 9-14. https://doi.org/10.1001/archpediatrics.2007.3

Yamaguchi, A., \& Knoblovits, P. (2018). Síndrome de Klinefelter y riesgo cardiovascular [Klinefelter syndrome and cardiovascular risk]. Hipertension y riesgo vascular, 35(4), 195-198. https://doi.org/10.1016/.hipert.2017.12.003 